International Mathematical Forum, 1, 2006, no. 25, 1195 - 1206

\title{
RESULTS CONCERNING AN EQUATION OF GOORMAGHTIGH AND RELATED TOPICS
}

\author{
Richard F. Ryan \\ Marymount College \\ Rancho Palos Verdes, CA 90275-6299 USA \\ rryan@marymountpv.edu
}

\begin{abstract}
Y. Bugeaud and T. N. Shorey [1] studied the equation $\frac{x^{u}-1}{x-1}=\frac{y^{w}-1}{y-1}$ in integers $y>x>1, u>1$, and $w>1$. They showed that, if you fix the values of $x$ and $y$, there are at most two solutions to this equation. The focus of the current paper is on exploring properties of the exponents if two solutions exist. The equation $\sigma(x) / x=\sigma\left(p^{m_{1}} q^{n_{1}}\right) /\left(p^{m_{1}} q^{n_{1}}\right)$ and relationships between $\left(x^{u}-1\right) /(x-1)=\left(y^{w}-1\right) /(y-1)$ and $\sigma\left(p^{m_{1}} q^{n_{1}}\right) /\left(p^{m_{1}} q^{n_{1}}\right)=\sigma\left(p^{m_{2}} q^{n_{2}}\right) /\left(p^{m_{2}} q^{n_{2}}\right)$ are also discussed, where $p$ and $q$ represent prime numbers, $m_{1}, n_{1}, m_{2}$, and $n_{2}$ are positive integers, and $p^{m_{1}} q^{n_{1}} \neq p^{m_{2}} q^{n_{2}}$.
\end{abstract}

Mathematics Subject Classification: 11D61. Secondary: 11A25.

Keywords: Equation of Goormaghtigh, abundancy.

\section{Introduction}

A well-known equation of Goormaghtigh is given by

$$
\frac{x^{u}-1}{x-1}=\frac{y^{w}-1}{y-1} \quad \text { in integers } x>1, y>1, u>2, w>2 \text {, with } x \neq y .
$$

We will assume that $x<y$ since doing so does not restrict the solutions in any meaningful way. Thus $u>w$. R. Goormaghtigh [3] noted that solutions to (1) are given by $(x, y, u, w)=(2,5,5,3)$ or $(2,90,13,3)$; it has been conjectured that these are the only solutions. A. Mạkowski and A. Schinzel [5] showed 
that $(2,5,5,3)$ is the only solution to (1) such that $y \leq 10$ or $u \leq 5$. Recently, Y. Bugeaud and T. N. Shorey [1] considered the equation

$$
\frac{x^{u}-1}{x-1}=\frac{y^{w}-1}{y-1} \quad \text { in integers } y>x>1, u>1, w>1 .
$$

They proved the following remarkable result.

Theorem 1.1. (Bugeaud and Shorey) Suppose that the values of $x$ and $y$ are fixed. If $\operatorname{gcd}(x, y)>1$ or if $y \geq 10^{11}$, then (2) has at most one solution. Further, if $y \geq 7$, then (2) has at most two solutions. Finally, the only solutions of (2) with $y \leq 6$ are given by $(x, y, u, w)=(2,5,5,3)$ or $(2,6,3,2)$.

We need to discuss some notation at this time. If $a$ and $s$ are integers, where $a>1$ and $s \geq 0$, we will let $Q_{s}(a)=\left(a^{s}-1\right) /(a-1)$. Obviously, if $s \geq 1$ then $\operatorname{gcd}\left(a, Q_{s}(a)\right)=1$. Let $p$ and $m$ represent a prime number and nonnegative integer, respectively. If $p^{m} \mid a$ (i.e., if $p^{m}$ divides $a$ ) but $p^{m+1} \nmid a$, we will write $v_{p}(a)=m$. It is easy to observe that, for each nonnegative integer $n, v_{p}\left(a^{n}\right)=n v_{p}(a)$. Though the following has become a corollary to theorem 1.1, its contrapositive was established in 1959 [5]. For the purpose of developing the current paper's results in a methodic way, it will be useful to present a variation of Mạkowski and Schinzel's proof.

Corollary 1.2. If, for given values of $x$ and $y$, there exist two distinct solutions of equation (2) then (the values of) $x$ and $y$ are relatively prime.

Proof. Assume that the values of $x$ and $y$ are fixed, say $x=a$ and $y=b$. Let $\left(a, b, u_{1}, w_{1}\right)$ and $\left(a, b, u_{2}, w_{2}\right)$ represent two distinct solutions of (2). Without loss of generality, assume that $u_{2}>u_{1}$. Obviously,

$$
Q_{u_{1}}(a)=Q_{w_{1}}(b)
$$

and

$$
Q_{u_{2}}(a)=Q_{w_{2}}(b)
$$

Subtracting 1 from both sides of (3), we get

$$
a \frac{a^{u_{1}-1}-1}{a-1}=b \frac{b^{w_{1}-1}-1}{b-1} .
$$

If there is a prime $q$ that divides $a$ and $b$ then

$$
v_{q}(a)=v_{q}(b)
$$


due to the fact that $\operatorname{gcd}\left(a, Q_{u_{1}-1}(a)\right)=\operatorname{gcd}\left(b, Q_{w_{1}-1}(b)\right)=1$. Subtracting (3) from (4) we see that

$$
a^{u_{1}} \frac{a^{u_{2}-u_{1}}-1}{a-1}=b^{w_{1}} \frac{b^{w_{2}-w_{1}}-1}{b-1}
$$

and so $u_{1} v_{q}(a)=w_{1} v_{q}(b)$. Dividing this equation by (6) we get $u_{1}=w_{1}$, which contradicts $u_{1}>w_{1}$. Thus $a$ and $b$ are relatively prime.

Formulas for $u_{2}$ and $w_{2}$, in terms of $u_{1}$ and $w_{1}$ respectively, will be presented in this paper. Before doing this, we need to review a few more known properties. When $w=2$, equation (2) can be written as

$$
x \frac{x^{u-1}-1}{x-1}=y \text {. }
$$

In this case $x$ divides $y$. M. Le [4] showed that, if $(x, y, u, 3)$ is a solution of (2) such that $(x, y, u) \notin\{(2,5,5),(2,90,13)\}$, then $\operatorname{gcd}(x, y)>1$ and $x \nmid y$. Consequently, if there is a solution of (2), other than $(2,5,5,3)$, such that $\mathrm{x}$ and $\mathrm{y}$ are relatively prime then $\mathrm{w} \geq 4$. Due to an aforementioned result of Mạkowski and Schinzel, $(2,5,5,3)$ is the only solution such that $(x, y)=(2,5)$. Thus, if $\left(a, b, u_{1}, w_{1}\right)$ and $\left(a, b, u_{2}, w_{2}\right)$ are distinct solutions of (2), then $u_{1}, u_{2} \geq 6$ and $w_{1}, w_{2} \geq 4$.

Each of the following four results is either a special case of, or is easily derived from, properties compiled by P. Ribenboim [6]; they will be useful throughout this paper. For these properties, we will let $a, u_{1}$, and $u_{2}$ represent integers, where $a>1$ and $u_{1}, u_{2} \geq 1$.

(1.3) $Q_{u_{1}}(a)$ divides $Q_{u_{2}}(a)$ if and only if $u_{1}$ divides $u_{2}$.

(1.4) If $g_{u}=\operatorname{gcd}\left(u_{1}, u_{2}\right)$ then $Q_{g_{u}}(a)=\operatorname{gcd}\left(Q_{u_{1}}(a), Q_{u_{2}}(a)\right)$.

(1.5) If $p$ is an odd prime dividing $a-1$ then $v_{p}\left(Q_{u_{1}}(a)\right)=v_{p}\left(u_{1}\right)$.

(1.6) If 4 divides $a-1$ then $v_{2}\left(Q_{u_{1}}(a)\right)=v_{2}\left(u_{1}\right)$.

At this time we will look at terminology and basic properties relevant to studying the other equations mentioned in this paper's abstract. Let a represent a positive integer, and $\sigma(a)$ denote the sum of the (positive) factors of $a$. The abundancy (or abundancy index) of $a$, denoted by $I(a)$, is defined by $I(a)=\sigma(a) / a$.

(1.7) If $p$ is prime and $m$ is a nonnegative integer then

$$
\sigma\left(p^{m}\right)=\sum_{j=0}^{m} p^{j}=\frac{p^{m+1}-1}{p-1} .
$$


(1.8) If $a=\prod_{j=1}^{k} p_{j}^{m_{j}}$, where $p_{1}, p_{2}, p_{3}, \ldots, p_{k}$ are distinct primes, $k$ is a positive integer, and $m_{1}, m_{2}, m_{3}, \ldots, m_{k}$ are nonnegative integers, then

$$
\sigma(a)=\prod_{j=1}^{k} \frac{p_{j}^{m_{j}+1}-1}{p_{j}-1} .
$$

Consequently, $\sigma$ is a multiplicative function; i.e., if $b$ and $c$ are relatively prime then $\sigma(b c)=\sigma(b) \sigma(c)$. Similarly,

$$
I(a)=\prod_{j=1}^{k} \frac{p_{j}^{m_{j}+1}-1}{p_{j}^{m_{j}}\left(p_{j}-1\right)}
$$

where $I$ is multiplicative.

In 1959, P. Erdős [2] studied the equation $I(x)=I(y)$. More recently, the current author [7] presented some results concerning the equation

$$
I(x)=I\left(p^{m_{1}} q^{n_{1}}\right)
$$

where the values of the distinct primes $p$ and $q$ were fixed; during this paragraph, assume that the positive integer values of $m_{1}$ and $n_{1}$ are also fixed. Obviously, $x=p^{m_{1}} q^{n_{1}}$ is a solution of (8). Giving a special case of an easily proven result: If $\sigma\left(p^{m_{1}} q^{n_{1}}\right)$ is relatively prime to $p q$, then $x=p^{m_{1}} q^{n_{1}}$ is the unique solution to (8). If $\left(\operatorname{gcd}\left(\sigma\left(p^{m_{1}} q^{n_{1}}\right), p q\right)>1\right.$, additional solutions exist in some instances. One possible form for additional solutions is $x=p^{m_{2}} q^{n_{2}}$, where $m_{2}$ and $n_{2}$ represent positive integers. It is easy to show that $m_{2} \neq m_{1}$; likewise, $n_{2} \neq n_{1}$. Relationships between (1) and $I\left(p^{m_{1}} q^{n_{1}}\right)=I\left(p^{m_{2}} q^{n_{2}}\right)$ will be discussed in this paper. A few more results concerning (8) will be presented at the end of this article; additional results regarding this equation will appear in a forthcoming paper.

\section{Main Results}

Theorem 2.1. Suppose that, for fixed values of $x$ and $y$, say $x=a$ and $y=$ $b$, there exist two distinct solutions of (2). Let $\left(a, b, u_{1}, w_{1}\right)$ and $\left(a, b, u_{2}, w_{2}\right)$ represent those solutions, where $u_{1}<u_{2}$. Then:

(A) $\left(u_{1}-1\right)\left|\left(u_{2}-1\right),\left(w_{1}-1\right)\right|\left(w_{2}-1\right)$, and another solution of (2) is given by $\left(a^{u_{1}-1}, b^{w_{1}-1}, \frac{u_{2}-1}{u_{1}-1}, \frac{w_{2}-1}{w_{1}-1}\right)$.

(B) Either $u_{1} \mid u_{2}$ and $w_{1} \mid w_{2}$, or $u_{2} \equiv 1\left(\bmod u_{1}\right)$ and $w_{2} \equiv 1\left(\bmod w_{1}\right)$. 
(C) If $u_{1} \mid u_{2}$ then $\left(a^{u_{1}}, b^{w_{1}}, \frac{u_{2}}{u_{1}}, \frac{w_{2}}{w_{1}}\right)$ is a solution of (2). Also, there exist positive integers $c$ and $d$ such that $u_{2}=c u_{1}\left(u_{1}-1\right)+u_{1}$ and $w_{2}=$ $d w_{1}\left(w_{1}-1\right)+w_{1}$, where $a^{u_{1}-1}\left|d w_{1}, b^{w_{1}-1}\right| c u_{1}$, and $\operatorname{gcd}\left(a, d w_{1} / a^{u_{1}-1}\right)=$ $\operatorname{gcd}\left(b, c u_{1} / b^{w_{1}-1}\right)=1$.

(D) If $u_{2} \equiv 1\left(\bmod u_{1}\right)$ then there exist positive integers $c$ and $d$ such that $u_{2}=c u_{1}\left(u_{1}-1\right)+1$ and $w_{2}=d w_{1}\left(w_{1}-1\right)+1$, where $a^{u_{1}-1} \mid\left(d w_{1}-1\right)$, $b^{w_{1}-1} \mid\left(c u_{1}-1\right)$, and $\operatorname{gcd}\left(a,\left(d w_{1}-1\right) / a^{u_{1}-1}\right)=\operatorname{gcd}\left(b,\left(c u_{1}-1\right) / b^{w_{1}-1}\right)=$ 1 .

(E) The only solutions $\left(a^{j}, b^{z}, u, w\right)$ to (2) such that $(j, z) \in\left\{\left(u_{1}-1, w_{1}-\right.\right.$ $\left.1),\left(u_{1}, w_{1}\right),\left(u_{2}-1, w_{2}-1\right),\left(u_{2}, w_{2}\right)\right\}$ are:

$\left(a^{u_{1}-1}, b^{w_{1}-1}, \frac{u_{2}-1}{u_{1}-1}, \frac{w_{2}-1}{w_{1}-1}\right)$ and $\left(a^{u_{1}}, b^{w_{1}}, \frac{u_{2}}{u_{1}}, \frac{w_{2}}{w_{1}}\right)$ if $u_{1} \mid u_{2}$, or

just $\left(a^{u_{1}-1}, b^{w_{1}-1}, \frac{u_{2}-1}{u_{1}-1}, \frac{w_{2}-1}{w_{1}-1}\right)$ if $u_{2} \equiv 1\left(\bmod u_{1}\right)$.

(F) The only solutions $\left(a^{j}, b^{z}, u, w\right)$ to (2) such that $j$ and $z$ are positive integers and $(j u, z w) \in\left\{\left(u_{1}-1, w_{1}-1\right),\left(u_{1}, w_{1}\right),\left(u_{2}-1, w_{2}-1\right),\left(u_{2}, w_{2}\right)\right\}$ are $\left(a, b, u_{1}, w_{1}\right),\left(a, b, u_{2}, w_{2}\right),\left(a^{u_{1}-1}, b^{w_{1}-1}, \frac{u_{2}-1}{u_{1}-1}, \frac{w_{2}-1}{w_{1}-1}\right)$ and, if $u_{1}$ divides $u_{2},\left(a^{u_{1}}, b^{w_{1}}, \frac{u_{2}}{u_{1}}, \frac{w_{2}}{w_{1}}\right)$.

Proof. Equations (3) through (5) and (7) hold true, and (4) implies that

$$
a \frac{a^{u_{2}-1}-1}{a-1}=b \frac{b^{w_{2}-1}-1}{b-1} .
$$

(A) Let $g_{U}=\operatorname{gcd}\left(u_{1}-1, u_{2}-1\right)$ and $g_{W}=\operatorname{gcd}\left(w_{1}-1, w_{2}-1\right)$. When the product of (5) and (9) is divided by $a b Q_{g_{U}}(a) Q_{g_{W}}(b)$, we get

$$
\frac{a^{u_{1}-1}-1}{a^{g_{U}}-1} \cdot \frac{b^{w_{2}-1}-1}{b^{g_{W}}-1}=\frac{a^{u_{2}-1}-1}{a^{g_{U}}-1} \cdot \frac{b^{w_{1}-1}-1}{b^{g_{W}}-1} .
$$

Since

$$
\operatorname{gcd}\left(\frac{a^{u_{1}-1}-1}{a^{g_{U}}-1}, \frac{a^{u_{2}-1}-1}{a^{g_{U}}-1}\right)=\operatorname{gcd}\left(\frac{b^{w_{1}-1}-1}{b^{g_{W}}-1}, \frac{b^{w_{2}-1}-1}{b^{g_{W}}-1}\right)=1,
$$

(10) implies that

$$
\frac{a^{u_{2}-1}-1}{a^{g_{U}}-1}=\frac{b^{w_{2}-1}-1}{b^{g_{W}}-1}
$$

Dividing (9) by (11) and adding 1 to both sides yields

$$
\frac{a^{g_{U}+1}-1}{a-1}=\frac{b^{g_{W}+1}-1}{b-1} .
$$


But $\left(a, b, g_{U}+1, g_{W}+1\right)$ cannot be a third solution of (2) such that $(x, y)=(a, b)$, due to theorem 1.1. Thus $g_{U}=u_{1}-1, g_{W}=w_{1}-1$, and so $\left(u_{1}-1\right) \mid\left(u_{2}-1\right)$ and $\left(w_{1}-1\right) \mid\left(w_{2}-1\right)$. Equation (3) implies that

$$
\frac{a^{u_{1}-1}-1 / a}{1-1 / a}=\frac{b^{w_{1}-1}-1 / b}{1-1 / b}<\frac{b^{w_{1}-1}}{1-1 / a} .
$$

Since $a^{u_{1}-1}$ cannot equal $b^{w_{1}-1}$, we see that $a^{u_{1}-1}<b^{w_{1}-1}$. Consequently, equation (11) implies that $\left(a^{u_{1}-1}, b^{w_{1}-1}, \frac{u_{2}-1}{u_{1}-1}, \frac{w_{2}-1}{w_{1}-1}\right)$ is a solution of (2).

(B) Due to the division algorithm, there exist unique integers $l_{u}, l_{w}, r_{u}$, and $r_{w}$ such that $u_{2}=l_{u} u_{1}+r_{u}$ and $w_{2}=l_{w} w_{1}+r_{w}$, where $l_{u}, l_{w}>0$, $0 \leq r_{u}<u_{1}$, and $0 \leq r_{w}<w_{1}$. Dividing (4) by (3) we get

$$
\frac{a^{l_{u} u_{1}+r_{u}}-1}{a^{u_{1}}-1}=\frac{b^{l_{w} w_{1}+r_{w}}-1}{b^{w_{1}}-1} .
$$

Thus

$$
a^{r_{u}} \frac{a^{l_{u} u_{1}}-1}{a^{u_{1}}-1}-b^{r_{w}} \frac{b^{l_{w} w_{1}}-1}{b^{w_{1}}-1}=\frac{b^{r_{w}}-1}{b^{w_{1}}-1}-\frac{a^{r_{u}}-1}{a^{u_{1}}-1} .
$$

Due to property 1.3, the left side of (12) is an integer. Also, the right side is (strictly) between -1 and 1 . So

$$
\frac{a^{r_{u}}-1}{a^{u_{1}}-1}=\frac{b^{r_{w}}-1}{b^{w_{1}}-1}
$$

Multiplying (3) with (13), we get $Q_{r_{u}}(a)=Q_{r_{w}}(b)$. But $\left(a, b, r_{u}, r_{w}\right)$ cannot be a third solution such that $(x, y)=(a, b)$. Thus $\left(r_{u}, r_{w}\right)=(0,0)$ or $(1,1)$, and so $u_{1} \mid u_{2}$ and $w_{1} \mid w_{2}$, or $u_{2} \equiv 1\left(\bmod u_{1}\right)$ and $w_{2} \equiv 1$ $\left(\bmod w_{1}\right)$.

(C) Assume that $u_{1} \mid u_{2}$. It is obvious that $a^{u_{1}-1}<b^{w_{1}-1}$, together with $a<b$, implies that $a^{u_{1}}<b^{w_{1}}$. Dividing (4) by (3) again, we see that $\left(a^{u_{1}}, b^{w_{1}}, \frac{u_{2}}{u_{1}}, \frac{w_{2}}{w_{1}}\right)$ is a solution of $(2)$. Since $u_{1}$ divides $u_{2}, u_{1}$ divides $u_{2}-u_{1}$. Obviously, $\left(u_{2}-1\right)-\left(u_{1}-1\right)=u_{2}-u_{1}$; it follows from part A of this theorem that $u_{1}-1$ divides $u_{2}-u_{1}$. So there exists a positive integer $c$ such that $u_{2}=c u_{1}\left(u_{1}-1\right)+u_{1}$. Similarly, there exists a positive integer $d$ such that $w_{2}=d w_{1}\left(w_{1}-1\right)+w_{1}$. When $(7)$ is divided by (5) we get

$$
a^{u_{1}-1} \frac{a^{c u_{1}\left(u_{1}-1\right)}-1}{a^{u_{1}-1}-1}=b^{w_{1}-1} \frac{b^{d w_{1}\left(w_{1}-1\right)}-1}{b^{w_{1}-1}-1} .
$$

Due to (5) and corollary $1.2, a \mid\left(b^{w_{1}-1}-1\right)$ and $b \mid\left(a^{u_{1}-1}-1\right)$. If 2 divides $a$ then $\left(b^{w_{1}-1}-1\right) /(b-1)$ is even, because of $(5)$, and $b-1$ is 
even. Consequently, if 2 divides $a$ then 4 divides $b^{w_{1}-1}-1$. So if $p_{0}$ is any prime divisor of $a$ then, due to (14) as well as properties 1.5 and 1.6, $v_{p_{0}}\left(a^{u_{1}-1}\right)=v_{p_{0}}\left(d w_{1}\right)$. It follows that $a^{u_{1}-1} \mid d w_{1}$, but $a$ and $d w_{1} / a^{u_{1}-1}$ are relatively prime. Similarly, $b^{w_{1}-1} \mid c u_{1}$, but $\operatorname{gcd}\left(b, c u_{1} / b^{w_{1}-1}\right)=1$.

(D) Assume that $u_{2} \equiv 1\left(\bmod u_{1}\right)$. Appealing to part A of this theorem once again, there exist positive integers $c$ and $d$ such that $u_{2}=c u_{1}\left(u_{1}-1\right)+1$ and $w_{2}=d w_{1}\left(w_{1}-1\right)+1$. When $(7)$ is divided by (5) we get

$$
a^{u_{1}-1} \frac{a^{\left(c u_{1}-1\right)\left(u_{1}-1\right)}-1}{a^{u_{1}-1}-1}=b^{w_{1}-1} \frac{b^{\left(d w_{1}-1\right)\left(w_{1}-1\right)}-1}{b^{w_{1}-1}-1} .
$$

It continues to be true that $a \mid\left(b^{w_{1}-1}-1\right)$, and if 2 divides $a$ then 4 divides $b^{w_{1}-1}-1$. So if $p_{0}$ is any prime divisor of $a$ then $v_{p_{0}}\left(a^{u_{1}-1}\right)=v_{p_{0}}\left(d w_{1}-1\right)$. It follows that $a^{u_{1}-1} \mid\left(d w_{1}-1\right)$, but $a$ and $\left(d w_{1}-1\right) / a^{u_{1}-1}$ are relatively prime. Similarly, $b^{w_{1}-1} \mid\left(c u_{1}-1\right)$, but $\operatorname{gcd}\left(b,\left(c u_{1}-1\right) / b^{w_{1}-1}\right)=1$.

(E) If there exists a solution such that $(x, y)=\left(a^{u_{1}-1}, b^{w_{1}-1}\right)$, other than $\left(a^{u_{1}-1}, b^{w_{1}-1}, \frac{u_{2}-1}{u_{1}-1}, \frac{w_{2}-1}{w_{1}-1}\right)$, it can be written as $\left(a^{u_{1}-1}, b^{w_{1}-1}, \frac{u_{3}-1}{u_{1}-1}, \frac{w_{3}-1}{w_{1}-1}\right)$ where $\left(u_{3}, w_{3}\right) \notin\left\{\left(u_{1}, w_{1}\right),\left(u_{2}, w_{2}\right)\right\}$. But then $\left(a, b, u_{3}, w_{3}\right)$ would be a third solution of $(2)$ such that $(x, y)=(a, b)$, which is not possible.

If there exists a solution that is not equal to $\left(a^{u_{1}}, b^{w_{1}}, \frac{u_{2}}{u_{1}}, \frac{w_{2}}{w_{1}}\right)$, such that $(x, y)=\left(a^{u_{1}}, b^{w_{1}}\right)$, it can be written in the form $\left(a^{u_{1}}, b^{w_{1}}, \frac{u_{3}}{u_{1}}, \frac{w_{3}}{w_{1}}\right)$ where $\left(u_{3}, w_{3}\right) \notin\left\{\left(u_{1}, w_{1}\right),\left(u_{2}, w_{2}\right)\right\}$. But then $\left(a, b, u_{3}, w_{3}\right)$ would be a third solution for $(x, y)=(a, b)$, and we have a contradiction.

If $\left(a^{u_{2}-1}, b^{w_{2}-1}, u_{3}, w_{3}\right)$ is a solution of $(2)$ then $\left(a^{u_{1}-1}, b^{w_{1}-1}, \frac{u_{3}\left(u_{2}-1\right)}{u_{1}-1}\right.$, $\left.\frac{w_{3}\left(w_{2}-1\right)}{w_{1}-1}\right)$ is a solution of $(2)$, different from $\left(a^{u_{1}-1}, b^{w_{1}-1}, \frac{u_{2}-1}{u_{1}-1}, \frac{w_{2}-1}{w_{1}-1}\right)$, and we have a contradiction. If $\left(a^{u_{2}}, b^{w_{2}}, u_{3}, w_{3}\right)$ is a solution of $(2)$ then $\left(a, b, u_{2} u_{3}, w_{2} w_{3}\right)$ is also a solution, another contradiction.

(F) The proof of this part uses techniques similar to those found in the proof of part (E), and may be obtained from the author.

At this time, we will discuss a couple of relationships between (2) and the last equation in this article's abstract. Suppose that $(x, y, u, w)=\left(p^{j}, q^{z}, u_{1}, w_{1}\right)$ is a solution of (2), where $p$ and $q$ represent prime numbers; $j$ and $z$ are positive integers. It is easy to show that $p \neq q$. The following results are easily verified.

(i) It turns out that $I\left(p^{j u_{1}-1} q^{z\left(w_{1}-1\right)-1}\right)=I\left(p^{j\left(u_{1}-1\right)-1} q^{z w_{1}-1}\right)$. 
(ii) Suppose that $\left(p^{j}, q^{z}, u_{2}, w_{2}\right)$ is another solution of $(2)$; without loss of generality, assume that $u_{1}<u_{2}$. Then the following sets represent four distinct solutions $\{x, y\}$ to $I(x)=I(y):\left\{p^{j u_{1}-1} q^{z\left(w_{1}-1\right)-1}, p^{j\left(u_{1}-1\right)-1} q^{z w_{1}-1}\right\}$, $\left\{p^{j u_{2}-1} q^{z\left(w_{2}-1\right)-1}, p^{j\left(u_{2}-1\right)-1} q^{z w_{2}-1}\right\},\left\{p^{j u_{2}-1} q^{z\left(w_{2}-w_{1}\right)-1}, p^{j\left(u_{2}-u_{1}\right)-1} q^{z w_{2}-1}\right\}$ and $\left\{p^{j\left(u_{2}-1\right)-1} q^{z\left(w_{2}-w_{1}\right)-1}, p^{j\left(u_{2}-u_{1}\right)-1} q^{z\left(w_{2}-1\right)-1}\right\}$.

The following theorem indicates that the last equation in the abstract, when satisfied, will yield at least one solution to (1), along with other results.

Theorem 2.2. Suppose that

$$
I\left(p^{m_{1}} q^{n_{1}}\right)=I\left(p^{m_{2}} q^{n_{2}}\right)
$$

where $p$ and $q$ represent prime numbers, $m_{1}, n_{1}, m_{2}$, and $n_{2}$ are positive integers, and $p^{m_{1}} q^{n_{1}} \neq p^{m_{2}} q^{n_{2}}$; thus $p \neq q$. Let $g=\operatorname{gcd}\left(m_{1}+1, m_{2}+1\right)$, $h=\operatorname{gcd}\left(n_{1}+1, n_{2}+1\right), k=\frac{m_{2}+1}{g}$, and $l=\frac{n_{1}+1}{h}$. Without loss of generality, we will assume that $p^{g}<q^{h}$ and $m_{1}<m_{2}$. Then:

(A) A solution of (1) is given by $(x, y, u, w)=\left(p^{g}, q^{h}, k, l\right)$.

(B) If $g=m_{2}-m_{1}$ (or equivalently, if $h=n_{1}-n_{2}$ ), then $m_{1}$ and $m_{2}$ are relatively prime; likewise for $n_{1}$ and $n_{2}$; additionally, $v_{2}\left(m_{1}+1\right) \neq$ $v_{2}\left(m_{2}+1\right)$ and $v_{2}\left(n_{1}+1\right) \neq v_{2}\left(n_{2}+1\right)$.

(C) Suppose that $g<m_{2}-m_{1}$. Then another solution to (1) is given by $\left(p^{g}, q^{h}, \frac{m_{2}-m_{1}}{g}, \frac{n_{1}-n_{2}}{h}\right)$. Also, $\left\{p^{m_{1}} q^{n_{1}}, p^{m_{2}} q^{n_{2}}\right\},\left\{p^{m_{2}-g} q^{n_{1}}, p^{m_{2}} q^{n_{1}-h}\right\}$, $\left\{p^{m_{1}} q^{n_{1}-h}, p^{m_{2}-g} q^{n_{2}}\right\}$, and $\left\{p^{m_{2}-m_{1}-g-1} q^{n_{1}-n_{2}-1}, p^{m_{2}-m_{1}-1} q^{n_{1}-n_{2}-h-1}\right\}$ represent four distinct solutions $\{x, y\}$ of $I(x)=I(y)$.

Proof. (A) If (15) is true then

$$
p^{m_{2}-m_{1}} \frac{p^{m_{1}+1}-1}{p^{g}-1} \cdot \frac{q^{n_{1}+1}-1}{q^{h}-1}=q^{n_{1}-n_{2}} \frac{p^{m_{2}+1}-1}{p^{g}-1} \cdot \frac{q^{n_{2}+1}-1}{q^{h}-1} .
$$

Note that $n_{1}>n_{2}$. Due to the fact that

$$
\operatorname{gcd}\left(\frac{p^{m_{1}+1}-1}{p^{g}-1}, \frac{p^{m_{2}+1}-1}{p^{g}-1}\right)=\operatorname{gcd}\left(\frac{q^{n_{1}+1}-1}{q^{h}-1}, \frac{q^{n_{2}+1}-1}{q^{h}-1}\right)=1
$$

we see that

$$
\frac{p^{m_{2}+1}-1}{p^{g}-1}=\frac{q^{n_{1}+1}-1}{q^{h}-1} .
$$

Thus $\left(p^{g}, q^{h}, k, l\right)$ is a solution of (1). 
(B) Equations (16) and (17) imply that

$$
p^{m_{2}-m_{1}} \frac{p^{m_{1}+1}-1}{p^{g}-1}=q^{n_{1}-n_{2}} \frac{q^{n_{2}+1}-1}{q^{h}-1} .
$$

Subtracting (18) from (17) we get

$$
\frac{p^{m_{2}-m_{1}}-1}{p^{g}-1}=\frac{q^{n_{1}-n_{2}}-1}{q^{h}-1} .
$$

It is easy to observe, from (19), that $g=m_{2}-m_{1}$ if and only if $h=n_{1}-n_{2}$. Now suppose that $g=m_{2}-m_{1}$. Since $m_{2}=g k-1, m_{2}$ is relatively prime to $g$. Thus $m_{1}$ and $m_{2}$ are relatively prime. If $v_{2}\left(m_{1}+1\right)=v_{2}\left(m_{2}+1\right)$, then $v_{2}(g)>v_{2}\left(m_{1}+1\right)$, which is impossible. Similarly, $n_{1}$ and $n_{2}$ are relatively prime and $v_{2}\left(n_{1}+1\right) \neq v_{2}\left(n_{2}+1\right)$.

(C) Assume that $g<m_{2}-m_{1}$. Due to (19), $\left(p^{g}, q^{h}, \frac{m_{2}-m_{1}}{g}, \frac{n_{1}-n_{2}}{h}\right)$ is a solution of (1). It follows from (ii) that the listed sets are distinct solutions of $I(x)=I(y)$.

The following observations concerning theorem 2.2 may be of interest.

(iii) If $\left\{p^{m_{1}} q^{n_{1}}, p^{m_{2}} q^{n_{2}}\right\} \neq\{80,200\}$ then $\left(p^{g}, q^{h}, k, l\right) \neq(2,5,5,3)$, which is easily established. It obviously follows that, if $\left\{p^{m_{1}} q^{n_{1}}, p^{m_{2}} q^{n_{2}}\right\} \neq$ $\{80,200\}$, then $k \geq 6$ and $l \geq 4$.

(iv) If $\left(p^{g}, q^{h}, k, l\right)$ is the only solution to (1) such that $(x, y)=\left(p^{g}, q^{h}\right)$ (for example, if $\left(p^{g}, q^{h}, k, l\right)$ is a solution such that $\left.q^{h}>10^{11}\right)$, then $g=m_{2}-m_{1}$.

At this point, the reader may be anticipating the following corollary.

Corollary 2.3. Suppose we are given positive even integers $m_{1}$ and $n_{1}$, and distinct odd primes $p$ and $q$. If $\max \{p, q\}>10^{11}$ then $x=p^{m_{1}} q^{n_{1}}$ is the only solution to (8) of the form $p^{m} q^{n}$, where $m$ and $n$ represent positive integers.

Proof. Due to premises of this corollary, $\sigma\left(p^{m_{1}} q^{n_{1}}\right)$ is odd. If there exist positive integers $m_{0}$ and $n_{0}$ such that $p^{m_{0}} q^{n_{0}}$ is a solution of (8), where $p^{m_{0}} q^{n_{0}} \neq$ $p^{m_{1}} q^{n_{1}}$, then $\sigma\left(p^{m_{0}} q^{n_{0}}\right)$ is odd too; thus $m_{0}$ is even. However, due to theorem $2.2(\mathrm{~B}), m_{0}$ and $m_{1}$ must be relatively prime, and we have a contradiction.

The following may be useful in cases where $p$ and $q$ are less than $10^{11}$. 
Corollary 2.4. Suppose we are given positive even integers $m_{1}$ and $n_{1}$, and distinct odd primes $p$ and $q$. Also suppose that, for each pair of divisors $m^{*}$ and $n^{*}$, where $m^{*}\left|\left(m_{1}+1\right), n^{*}\right|\left(n_{1}+1\right), m^{*} \neq m_{1}+1, n^{*} \neq n_{1}+1$, and $\max \left\{p^{m^{*}}, q^{n^{*}}\right\}<10^{11}$, there is at most one solution of (2) such that $(x, y)=$ $\left(\min \left\{p^{m^{*}}, q^{n^{*}}\right\}, \max \left\{p^{m^{*}}, q^{n^{*}}\right\}\right)$. Then $p^{m_{1}} q^{n_{1}}$ is the only solution to (8) of the form $p^{m} q^{n}$, where $m$ and $n$ represent positive integers.

The proof of corollary 2.3 suffices as a sketch of the proof for corollary 2.4. As a result of the following theorem, when the values of $p, q, m_{1}$, and $n_{1}$ are given, equation (8) has a finite number of solutions of the form $p^{m} q^{n}$. Note that $\tau(n)$ denotes the number of positive divisors of $n$.

Theorem 2.5. Suppose we are given positive integers $m_{1}$ and $n_{1}$, and distinct primes $p$ and $q$. Then the number of solutions to (8) of the form $p^{m} q^{n}$, where $m$ and $n$ continue to represent positive integers, is less than or equal to $\min \left\{\tau\left(m_{1}+1\right), \tau\left(n_{1}+1\right)\right\}$.

Partial proof. Let $t=\min \left\{\tau\left(m_{1}+1\right), \tau\left(n_{1}+1\right)\right\}$. First, consider the case in which $t=\tau\left(m_{1}+1\right)$. Suppose there are $t+1$ distinct solutions in the specified form. We will denote those solutions by $p^{m_{1}} q^{n_{1}}, p^{m_{2}} q^{n_{2}}, \ldots, p^{m_{t+1}} q^{n_{t+1}}$. (In contrast to theorem 2.2, we are not assuming that $m_{1}<m_{2}$.) Since $\operatorname{gcd}\left(m_{1}+\right.$ $\left.1, m_{i}+1\right)<m_{1}+1$ for all $i \in\{2,3, \ldots, t+1\}$, there exist $\alpha, \beta \in\{2,3, \ldots, t+1\}$, where $\alpha \neq \beta$, such that $\operatorname{gcd}\left(m_{1}+1, m_{\alpha}+1\right)=\operatorname{gcd}\left(m_{1}+1, m_{\beta}+1\right)$. There are several cases to consider. If $m_{1}=\max \left\{m_{1}, m_{\alpha}, m_{\beta}\right\}$ then

$$
\frac{q^{n_{\alpha}+1}-1}{q^{h_{\alpha}}-1}=\frac{q^{n_{\beta}+1}-1}{q^{h_{\beta}}-1}
$$

where $h_{\alpha}=\operatorname{gcd}\left(n_{1}+1, n_{\alpha}+1\right)$ and $h_{\beta}=\operatorname{gcd}\left(n_{1}+1, n_{\beta}+1\right)$. It follows that $h_{\alpha}=h_{\beta}, n_{\alpha}=n_{\beta}, I\left(p^{m_{\alpha}}\right)=I\left(p^{m_{\beta}}\right)$, and finally $m_{\alpha}=m_{\beta}$. So the distinct solutions $p^{m_{\alpha}} q^{n_{\alpha}}$ and $p^{m_{\beta}} q^{n_{\beta}}$ are equal, and we have a contradiction. The situations in which $m_{1} \neq \max \left\{m_{1}, m_{\alpha}, m_{\beta}\right\}$ can be addressed in corresponding fashions. Details may be obtained from the author. When $t=\tau\left(n_{1}+1\right)$, the proof is obviously similar to the case in which $t=\tau\left(m_{1}+1\right)$.

Thus, if the hypothesis of theorem 2.5 is satisfied, and $m_{1}+1$ or $n_{1}+1$ is prime, then (8) has at most two distinct solutions in the specified form. The proof of the following statement, though not difficult, makes use of primitive factors for numbers in the form $q^{N}-1$ : If $p^{m_{1}} q^{n_{1}}, p^{m_{2}} q^{n_{2}}, \ldots, p^{m_{K}} q^{n_{K}}$ represent distinct solutions of (8), where $K \geq 3$ and $m_{1}, m_{2}, \ldots m_{K}, n_{1}, n_{2}, \ldots n_{K}$ are positive integers, then for all distinct $\alpha, \beta, \gamma \in\{1,2, \ldots, K\}, \operatorname{gcd}\left(m_{\alpha}+1, m_{\beta}+\right.$ 1) $\nmid \operatorname{gcd}\left(m_{\alpha}+1, m_{\gamma}+1\right)$; likewise, $\operatorname{gcd}\left(n_{\alpha}+1, n_{\beta}+1\right) \nmid \operatorname{gcd}\left(n_{\alpha}+1, n_{\gamma}+1\right)$. This result can be used to improve on the conclusion of theorem 2.5 for cases in which $m_{1}+1$ and $n_{1}+1$ may be composite numbers. For example, if the 
hypothesis of theorem 2.5 is satisfied, and $m_{1}+1$ or $n_{1}+1$ is any prime power, then (8) has a maximum of two distinct solutions in the specified form.

The present author tends to believe that $\left\{p^{m_{1}} q^{n_{1}}, p^{m_{2}} q^{n_{2}}\right\}=\{80,200\}$ is the only solution of (15), but is unable to prove this at the present time. As previously indicated, a well-known conjecture states that $\{(2,5,5,3),(2,90,13,3)\}$ is the solution set of (1). The following corollary to theorem 2.2 presents a relationship between a weaker version of this conjecture and the assertion in the first sentence of this paragraph.

Corollary 2.6. The set $\{80,200\}$ is the only solution of (15) if and only if $(2,5,5,3)$ is the only solution of (2) such that $(x, y)$ is of the form $\left(p^{j}, q^{z}\right)$, where $p$ and $q$ are prime numbers, and $j$ and $z$ are positive integers.

Proof. Suppose that $(2,5,5,3)$ is the only solution of $(2)$ such that $x$ and $y$ have the specified form. Without loss of generality, we will assume that $m_{1}<m_{2}$ in this proof. If (15) holds true then, due to theorem $2.2(\mathrm{~A}),\left(p^{g}, q^{h}, k, l\right)=$ $(2,5,5,3)$ where $g, h, k$, and $l$ are defined as they were in theorem 2.2. It follows from observation (iii) that $\left\{p^{m_{1}} q^{n_{1}}, p^{m_{2}} q^{n_{2}}\right\}$ must equal $\{80,200\}$.

Now assume that $\{80,200\}$ is the sole solution of $(15)$. If $\left(p^{j}, q^{z}, u_{1}, w_{1}\right)$ is a solution of $(2)$ then $\{80,200\}=\left\{p^{j u_{1}-1} q^{z\left(w_{1}-1\right)-1}, p^{j\left(u_{1}-1\right)-1} q^{z w_{1}-1}\right\}$ as a result of (i). It easily follows that $\left(p^{j}, q^{z}, u_{1}, w_{1}\right)=(2,5,5,3)$.

Let's conclude this article by noting that $I\left(2^{3} \cdot 5\right)=I\left(2^{5} \cdot 5^{\log _{5} 7}\right)=\frac{9}{4}$, where $\log _{5} 7$ is not an integer. Since $5^{\log _{5} 7}=7$, this is an (previously known) example in which $I\left(p^{m_{1}} q_{1}{ }^{n_{1}}\right)=I\left(p^{m_{2}} q_{2}{ }^{n_{2}}\right)$ where $p, q_{1}$, and $q_{2}$ are distinct primes, and $m_{1}, m_{2}, n_{1}$, and $n_{2}$ represent positive integers. This equation looks like an interesting topic for another time; however, I cannot resist pointing out that each pair of distinct even perfect numbers satisfies this equation. The following sentence is true for all positive integers $m_{1}, m_{2}, n_{1}$, and $n_{2}$, due to remarks made in a previous paper [7]: If $p_{1}, p_{2}, q_{1}$, and $q_{2}$ are distinct primes then $I\left(p_{1}{ }^{m_{1}} q_{1}{ }^{n_{1}}\right) \neq I\left(p_{2}{ }^{m_{2}} q_{2}{ }^{n_{2}}\right)$.

\section{References}

[1] Y. Bugeaud and T. N. Shorey, On the Diophantine equation $\frac{x^{m}-1}{x-1}=\frac{y^{n}-1}{y-1}$, Pacific J. Math., 207 (2002), 61 - 75.

[2] P. Erdős, Remarks on number theory II. Some problems on the $\sigma$ function, Acta Arith., 5 (1959), 171 - 177.

[3] R. Goormaghtigh, L'Intermédiaire des Mathématiciens, 24 (1917), 88.

[4] M. Le, Exceptional solutions of the exponential Diophantine equation $\left(x^{3}-1\right) /(x-1)=\left(y^{n}-1\right) /(y-1)$, J. Reine Angew. Math., 543 (2002), 187 - 192. 
[5] A. Mąkowski and A. Schinzel, Sur l'équation indéterminée de R. Goormaghtigh, Mathesis 68 (1959), 128 - 142.

[6] P. Ribenboim, Fermat's Last Theorem for Amateurs, Springer-Verlag, New York, 1999, pp. 80 - 91.

[7] R. F. Ryan, Results concerning uniqueness for $\sigma(x) / x=\sigma\left(p^{n} q^{m}\right) /\left(p^{n} q^{m}\right)$ and related topics, Int. Math. J., 2 (2002), 497 - 514.

Received: October 31, 2005 